\title{
Non-invasive Imaging of Sense of Smell by Tracking the Voltage-Gated Sodium Channel Nav1.7
}

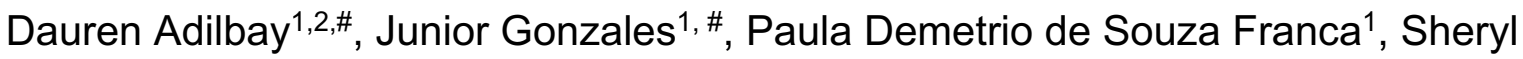
Roberts $^{1}$, Snehal Patel ${ }^{2}$, Tara Viray ${ }^{1}$, Chun Yuen Chow ${ }^{3,4}$, Glenn F. King ${ }^{3,4}$, Sanjay K. Jain $^{5,6,7}$, Alvaro A. Ordonez $z^{5,6}$, Laurence S. Carroll 5,7, Nagavarakishore Pillarsetty ${ }^{1,8, \neq, *}$, Thomas Reiner ${ }^{1,9, \neq, * *}$

${ }^{1}$ Department of Radiology, Memorial Sloan Kettering Cancer Center, New York, NY, USA

${ }^{2}$ Department of Surgery, Memorial Sloan Kettering Cancer Center, New York, NY, USA. ${ }^{3}$ Institute for Molecular Bioscience, The University of Queensland, St Lucia, QLD 4072, Australia

${ }^{4}$ Australian Research Council Centre of Excellence for Innovations in Peptide and Protein Science, The University of Queensland, St Lucia, QLD 4072, Australia ${ }^{5}$ Center for Infection and Inflammation Imaging Research, Johns Hopkins University School of Medicine, Baltimore, MD, USA

${ }^{6}$ Department of Pediatrics, Johns Hopkins University School of Medicine, Baltimore, MD, USA

${ }^{7}$ Russell H. Morgan Department of Radiology and Radiological Sciences, Johns Hopkins University School of Medicine, Baltimore, MD, USA

${ }^{8}$ Department of Radiology, Weill Cornell Medical College, New York, NY, USA

${ }^{9}$ Chemical Biology Program, Memorial Sloan Kettering Cancer Center, New York, NY, USA

\# Contributed equally

${ }^{\ddagger}$ Contributed equally 


\section{${ }^{*}$ Corresponding authors:}

Thomas Reiner

Department of Radiology and Chemical

Biology Program, Memorial Sloan Kettering Cancer Center,

New York 10065, United States

Phone: 6468883461

Email: reinert@mskcc.org

Nagavarakishore Pillarsetty

Department of Radiology,

Memorial Sloan Kettering Cancer Center, New York, NY, USA

1275 York Avenue, New York, NY 10065, USA.

Email: pillarsn@mskcc.org 


\section{Abstract}

Background: Anosmia/hyposmia affects 13.3 million people in the U.S. alone according to the recent U.S. National Health and Nutrition Examination Survey (NHANES). Hundreds of thousands more people with persistent olfactory dysfunction will be added to this number due to the COVID-19 pandemic. Patients with loss-offunction mutations in $S C N 9 A$, the gene encoding Nav1.7, experience anosmia in addition to congenital insensitivity to pain. Tsp1a is a recently discovered peptide that inhibits Nav1.7 with high potency and selectivity. In this study, we examined whether a fluorescently tagged version of Tsp1a could be used to visualize normal and damaged mouse olfactory nerves.

Methods: Athymic nude mice were intravenously injected with Tsp1a-IR800. As a control, mice were injected with PBS only, and as a blocking control were injected with combination of Tsp1a and Tsp1a-IR800. All mice were imaged in-vivo and epifluorescence images were acquired using an IVIS Spectrum animal imaging system. Semiquantitative analysis of the Tsp1a-IR800 signal was conducted by measuring the average radiant efficiency in the region of the olfactory epithelium/bulb (ROEB). Methimazole was used to chemically ablate the olfactory epithelium. We performed a food buried test to correlate the level of anosmia with the level of radiance efficiency.

Results: The area of olfactory epithelium/bulb was clearly visible in epifluorescence invivo images of mice receiving the imaging agent. The radiant efficiency was significantly less in both mice injected with PBS and in mice injected with the blocking formulation. The mice after olfactory ablation had a significantly reduced radiant efficiency compared 
with normal mice. Moreover, there was a statistically significant and inverse correlation between the time required for the mouse to find buried food and the radiant efficiency. We also performed immunohistochemistry using Nav1.7 antibody. Mice after olfactory ablation as well as COVID-19-infected mice had significantly lower expression of Nav1.7 on the level of olfactory epithelium/bulb.

Conclusion: We show that the fluorescent imaging of mouse olfactory epithelium/bulb is possible, suggesting that labeled Tsp1a tracers may serve as the first objective diagnostic tool of smell disorders, including those caused by COVID-19. 


\section{Introduction}

The classical five senses - sight, smell, touch, hearing and taste have evolved primarily to aid organisms with acquisition of food and nutrients, and to avoid toxins and harmful situations. The sense of smell — olfaction — is critical for survival of many animals because smell can convey information about food and the environment, and it serves as as a tool for inter- and intra-species communication. The types and dynamic ranges of smells detected by animals is vast. For example, it is estimated that the human nose can detect up to 1 trillion different scents [1], some of them at concentrations of less than one part per billion [2]. This range of detection is accomplished by a relatively small number ( 350$)$ of distinct olfactory receptors on olfactory sensory neurons (OSNs) that trigger ion channels and act as signal transducers [3]. Despite its fundamental importance in biology, the perception of olfaction, like other senses, is subjectively reported and is therefore a non-independent measure. The current standard of care lacks a subject-independent method/metric to measure the subject's ability to sense smell.

The latest epidemiological data from the U.S. National Health and Nutrition Examination Survey (HNANES) included a chemosensory protocol in collaboration with National Institute on Deafness and Other Communications Disorders (NIDCD) [4]. A staggering 13.3 million adults were found to have smell disorders, including 3.4 million with severe hyposmia/anosmia. While many reasons have been attributed to the loss of olfaction, one cause is mutations in ion channels that transmit messages from olfactory neurons 
to the brain. In particular, the voltage-gated sodium channel 1.7 (Nav1.7) plays a critical role in olfaction among mammals [5]. Nav1.7 is essential for perceiving pain but it also plays important role in olfaction. We recently isolated Tsp1a, a potent and subtypeselective peptidic inhibitor of Nav1.7, from the venom of a Peruvian tarantula [6, 7]. We showed that a fluorescently labeled version of synthetic Tsp1a peptide can be used to identify mouse peripheral nerves in vivo without any side-effects, making it a potentially clinically translatable imaging agent.

Clinically, olfaction disorders are a neglected group of diseases with episodic attention from the scientific community [8]. Despite low levels of self-reporting, and presumed low priority, it has a tremendous impact on the quality of life and public health [4]. Several studies revealed that individuals with olfactory disorders experience taste problems, related decrease in appetite, difficulty with cooking, and difficulties with social interaction and maintaining personal hygiene [9-11]. The detection of hazardous odors was also impaired, resulting in a sense of fear and frustration [9]. Overall, the influence on the quality of life can be devastating, with more than $20 \%$ of patients experiencing symptoms of depression $[12,13]$. Here we explore the utility of fluorescent Tsp1a in detecting Nav1.7-related olfaction disorders.

Nav1.7 is expressed predominantly in peripheral sensory neurons and the axons of human olfactory sensory neurons (OSNs). In OSNs, Nav1.7 is critical for transmitting olfactory cues to higher-order neurons in the brain. Therefore, in addition to mutations in the SCN9A gene encoding Nav1.7, any insults (e.g., viral infections or inflammation 
resulting from allergies or drugs), that result in loss or interference with the normal function of the superficial layer of the ROEB can lead to loss of odorant perception. The critical role of Nav1.7 in the superficial layer of the ROEB might provide a means for stratification of patients with respect to their sense of smell. In this work, we report the characterization of a fluorescently labeled molecular probe (Tsp1a-IR800) that is specific for Nav1.7 and can function as a marker of olfaction. We confirm previous studies showing that Nav1.7 is abundantly expressed in the nasal epithelium, and show that Tsp1a-IR800 can be used to non-invasively image Nav1.7 on olfactory epithelium using epifluorescence imaging in vivo. We also demonstrate that chemically-induced anosmia in mice from methimazole treatment results in loss of sustentacular cells and concomitant diminution of Nav1.7 expression in OSNs. This is without a significant global reduction in Nav1.7 expression detected by our imaging probe Tsp1a-IR800. The presence of anosmia in methimazole-treated mice was confirmed by a buried food test and the time for food detection. This was inversely correlated to fluorescence intensity in olfactory epithelium. In the light of current COVID-19 pandemic, we also demonstrate that SARS-COV2-infected mice have diminished Nav1.7 expression in the olfactory epithelium/bulb, confirming that dysfunction of olfactory system provokes deprivation of smell. Together, our findings demonstrate that fluorescently labeled Tsp1a targeting Nav1.7 channels can serve as a non-invasive imaging tool for smell sensing.

\section{Results}

\section{Nav1.7 is abundantly expressed in the olfactory bulb/epithelium of normal mice}

We isolated and dissected olfactory bulb and epithelium regions from normal nude mice, and used immunohistochemistry (H\&E and Nav1.7 antibody staining) to identify 
areas of high Nav1.7 expression. Consistent with previous reports [5, 14], we found that Nav1.7 is moderately expressed in layers of primary OSNs, and highly expressed in olfactory nerve bundles located in lamina propria, including all the paths towards the olfactory bulb (Figure 1b). Olfactory bulbs abundantly express Nav1.7 on peripheral areas, which corresponds to the olfactory nerve layer (ONL). The deeper, glomerular layer located just after the ONL corresponds to terminal synapses of OSN axons with the dendrites of mitral and tufted cells, which are lightly stained (Figure 1b). 
a) Normal olfactory nerve stained with $H \& E$

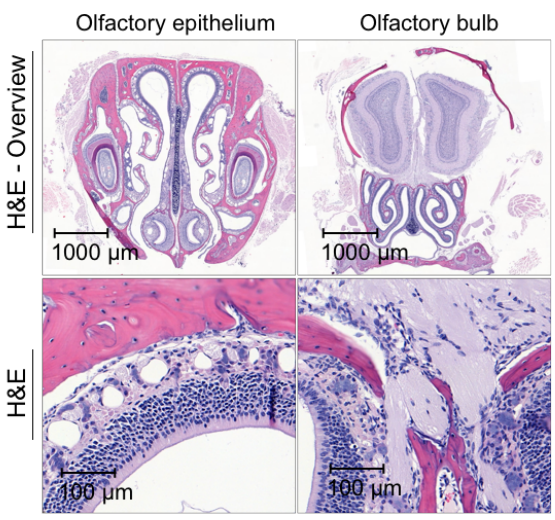

c) After olfactory ablation using methimazole

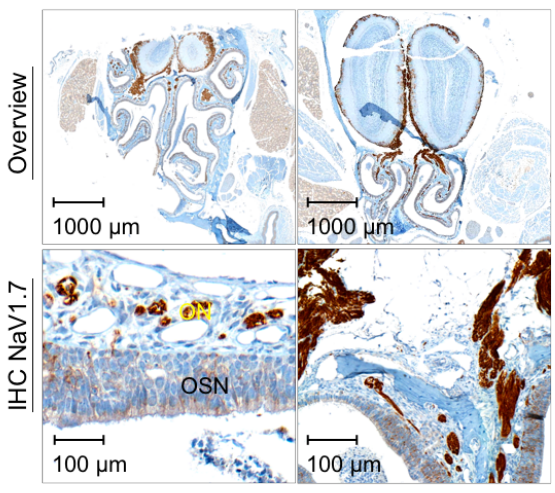

e) COVID-19 infected mice

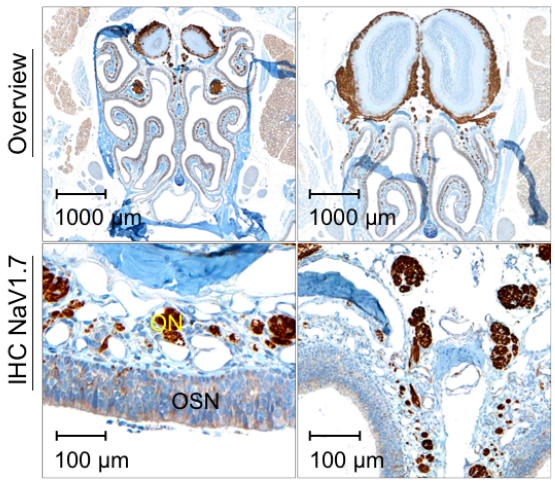

b) Normal olfactory nerve Nav1.7 IHC slides

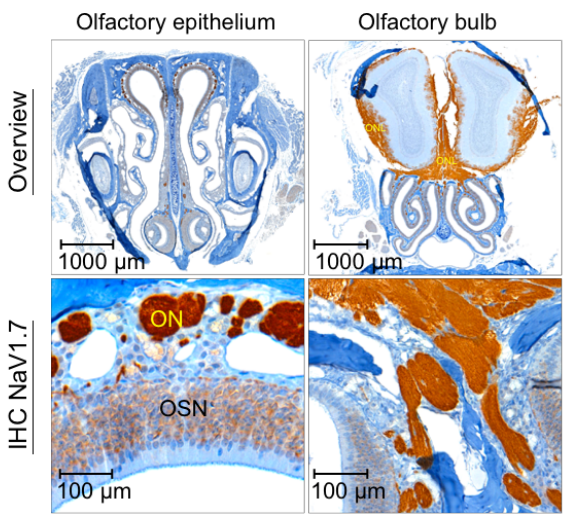

d) IGG control

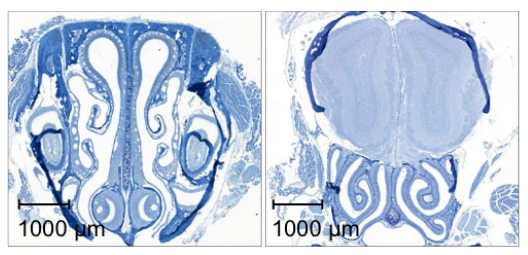

f) Quantification of Nav1.7 expression in olfactory epithelium

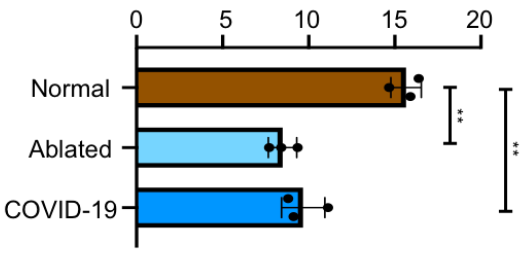

g) Quantification of Nav1.7 expression in olfactory bulb

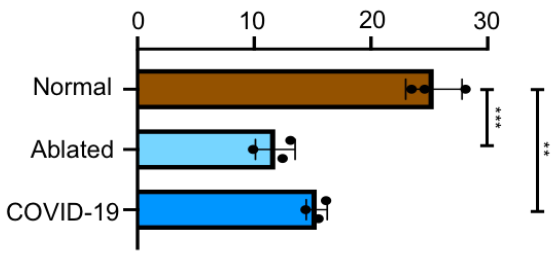

Figure 1. Histological slides of the olfactory bulb and olfactory epithelium of wild-type (WT) mice, mice with olfactory ablation, and a mouse infected with COVID-19. (a) H\&E stain of WT mouse. (b) Nav1.7 IHC slide of WT mouse. (c) IHC slide of the mouse after olfactory ablation. (d) IGG control slide. (e) IHC slides of a mouse with COVID-19 infection. f) Quantification of Nav1.7 expression in the olfactory epithelium. (g) Quantification of Nav1.7 expression in olfactory bulb. ${ }^{* *} \mathrm{P} \leq 0.01,{ }^{* * *} \mathrm{P} \leq 0.001 \mathrm{ON}-$ olfactory nerve bundles, OSN - olfactory sensory neurons, ONL - olfactory nerve layer. 
Nav1.7 expression is reduced in anosmia induced mouse model and COVID-19 infected mice models. The expression of Nav1.7 was significantly diminished after olfactory ablation using methimazole and also in a COVID-19 infected mouse (Figure 1c, e). Of the total tissue area in the olfactory epithelium of WT mice, $15.7 \%$ was Nav1.7-positive area, compared to $8.5 \%$ in olfactory-ablated mice and $9.7 \%$ in COVID19 infected mice (Figure 1f). The ONL of the olfactory bulb also showed a decrease in Nav1.7 expression in both olfactory-ablated and COVID-19 infected mice (Figure 1g).

Tsp1a-IR800 accumulates in olfactory nerves and ROEB. The area of the olfactory epithelium/bulb was clearly visible using fluorescence. Epifluorescence in-vivo images in mice receiving intravenous injection with the imaging agent Tsp1a-800 generated high contrast between the ROEB and its surrounding regions. The radiant efficiency was significantly less in both mice injected with PBS and in mice injected with the unmodified peptide (blocking agent) in combination with imaging agent (Figure 2b). We observed a 150-fold increase in radiant efficiency compared to mice injected with PBS and a 61 -fold increase compared to mice treated with blocking formulation. 
a)

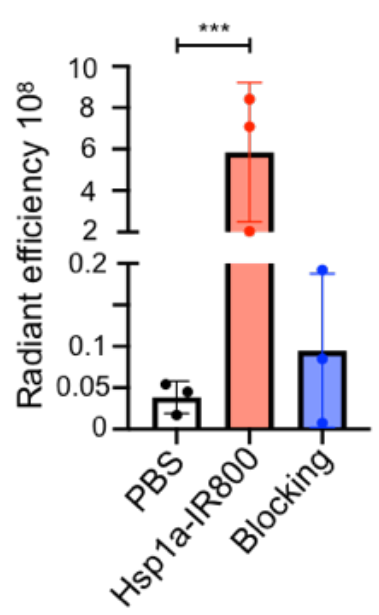

c)

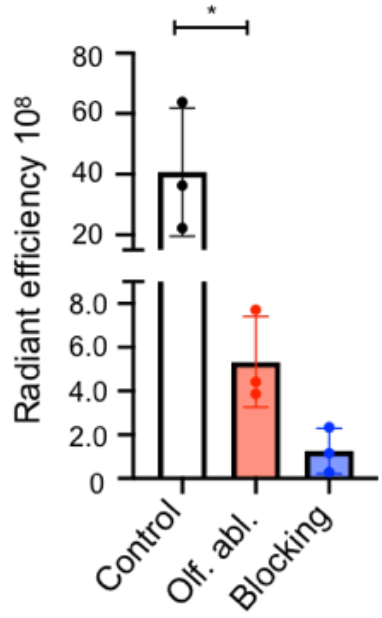

b)

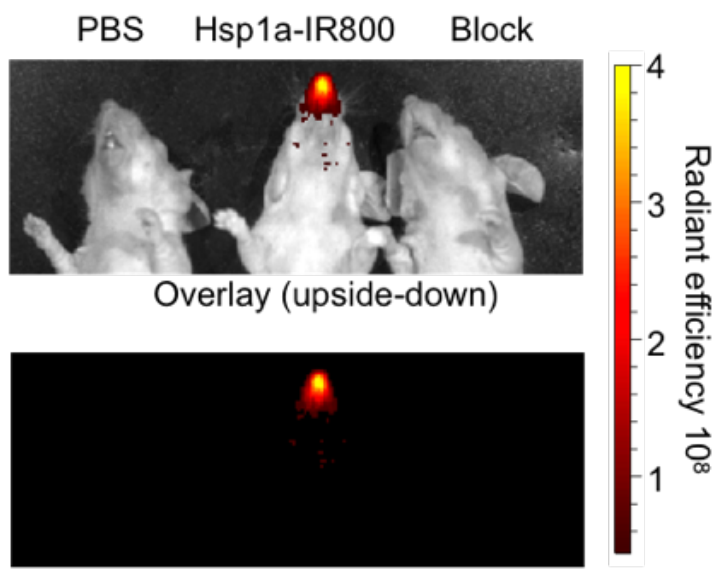

Fluorescence (upside-down)

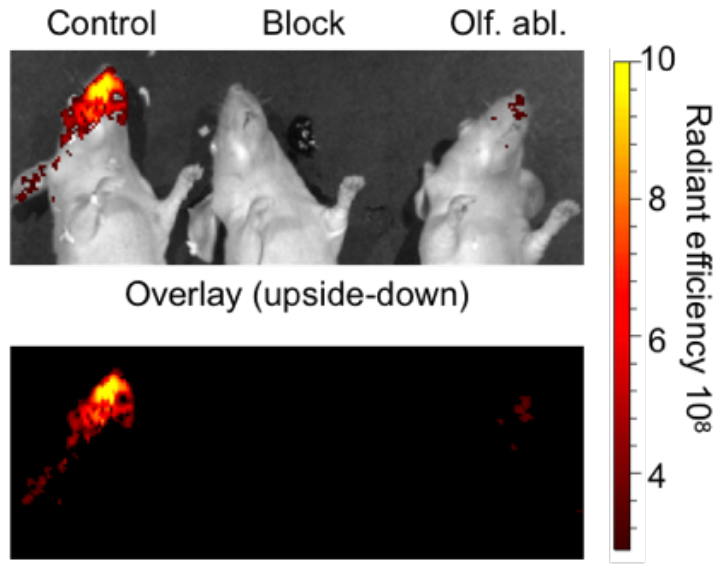

Fluorescence (upside-down)

Figure 2. Tsp1a-IR800 accumulation in the olfactory epithelium/bulb in WT mice and mice with olfactory ablation. (a) Fluorescence intensity quantification of panel b; (b) Epifluorescence images of animals injected with PBS, Tsp1a-IR800, and Tsp1aIR800/Tsp1a formulation respectively. Images were taken 30 min after tail vein injection; (c) Fluorescence intensity quantification of panel d; (d) Epifluorescence images of animals; control - WT mice injected with Tsp1a-IR800; block - WT mice injected with Tsp1a-IR800/Tsp1a; and olfactory ablation - mice after olfactory ablation using methimazole and injection of Tsp1a-IR800. Images were taken 30 min after tail vein injection. 
Additionally, we resected the olfactory epithelium region of these mice and obtained regular fluorescent microscopy images (Figure 3). The fluorescent microscopy images confirm the results of in-vivo epifluorescence imaging and IHC. OSN and olfactory nerve bundles located in the lamina propria showed the most intense fluorescence. WT mice and mice treated with blocking agent were negative for any signal in the red channel, while only nuclear-associated blue channel signal was visible corresponding to Hoechst.
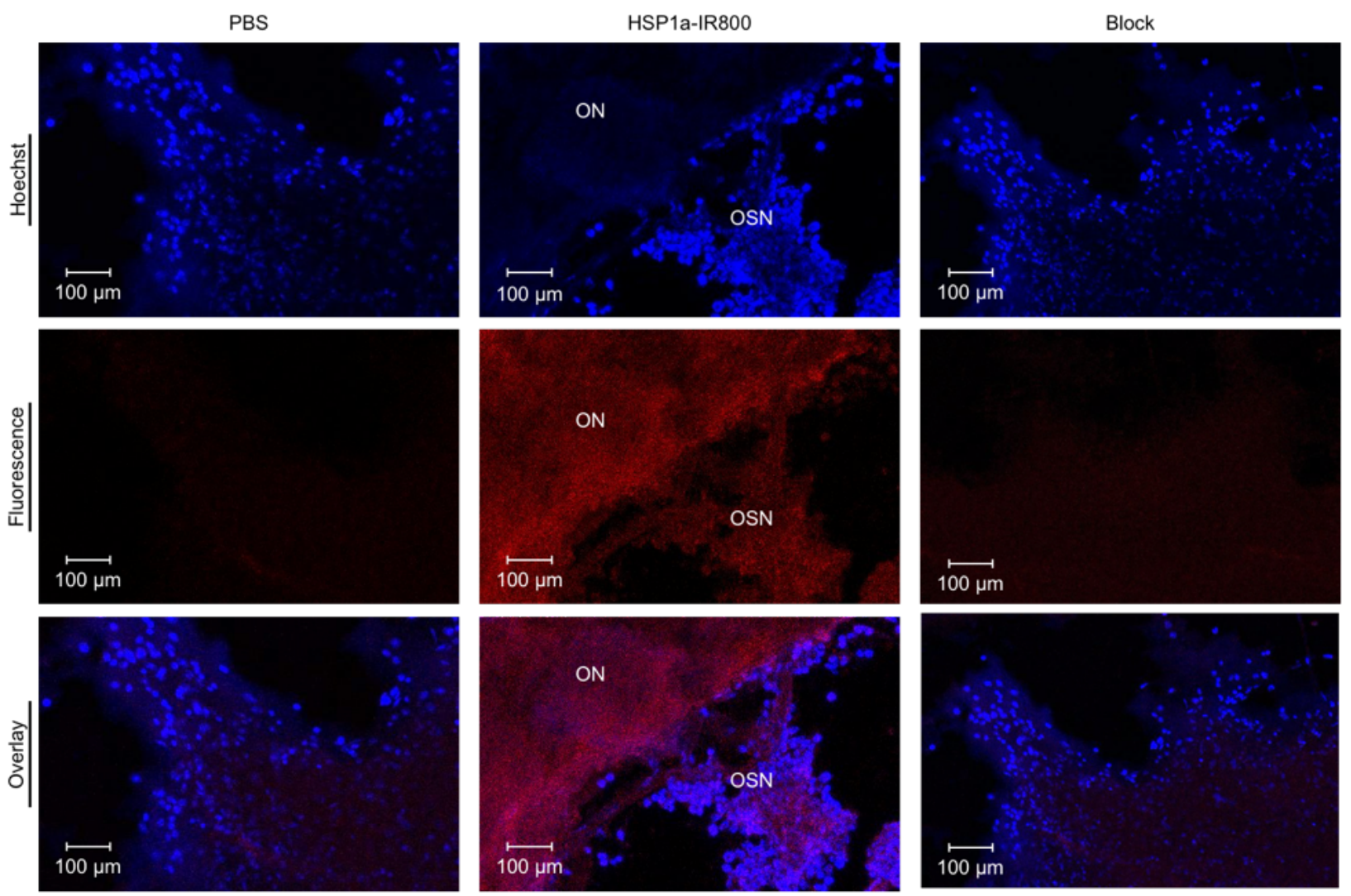

Figure 3. Fluorescent confocal microscopy images of the olfactory epithelium of animals injected with PBS, Tsp1a-IR800, and Tsp1a-IR800/Tsp1a formulation respectively. ON - olfactory nerve (bundles), OSN - olfactory sensory neurons.

\section{Olfactory ablation reduces the accumulation of Tsp1a-IR800.}

Mice with damaged olfactory nerves had visibly diminished radiant efficiency compared to WT mice. The olfactory bulb/epithelium region of methimazole-treated mice had a 
statistically significant 8-fold decrease in radiant efficiency compared to WT mice

(Figure 2c). The average radiant efficiency of the olfactory region of WT mice imaged from both sides were 4.08E+09 (s.d. 2.11E+09), compared to 5.33E+08 (s.d. 2.08E+08) for mice with an olfactory ablation (unpaired $t$-test, $\mathrm{p}=0.045$ ). Ex vivo images showed a similar statistically significant difference between mouse groups in the olfactory epithelium/bulb region. The heart and kidneys are the only internal organs with higher fluorescence signals compared to WT mice and mice treated with blocking agent.

\section{Time in food buried test correlates with the radiant efficiency of olfactory bulb/epithelium region of WT and olfactory-ablated mice.}

Mice with olfactory ablation needed a significantly longer time than WT mice to find buried food (Figure 4b). WT mice were able to find buried food in less than $30 \mathrm{~s}$, compared with a mean of $135 \mathrm{~s}$ for olfactory-ablated mice $(p<0.001)$. Furthermore, there was an inverse correlation between the Tsp1a-IR800 radiant efficiency and the time required to find buried food (Pearson correlation coefficient $r=-0.79, n=10, p=0.0056$; Figure 4c). 
a)

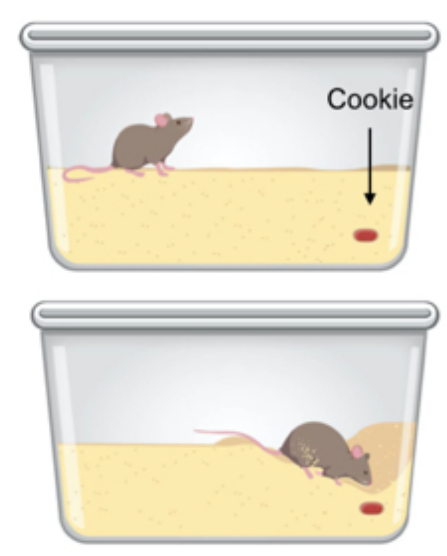

b)

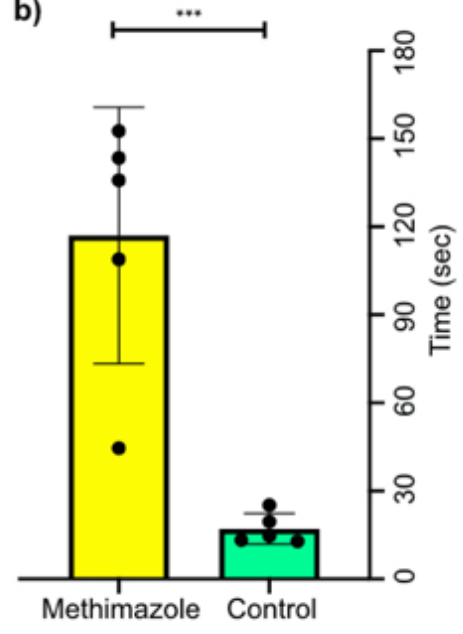

c)

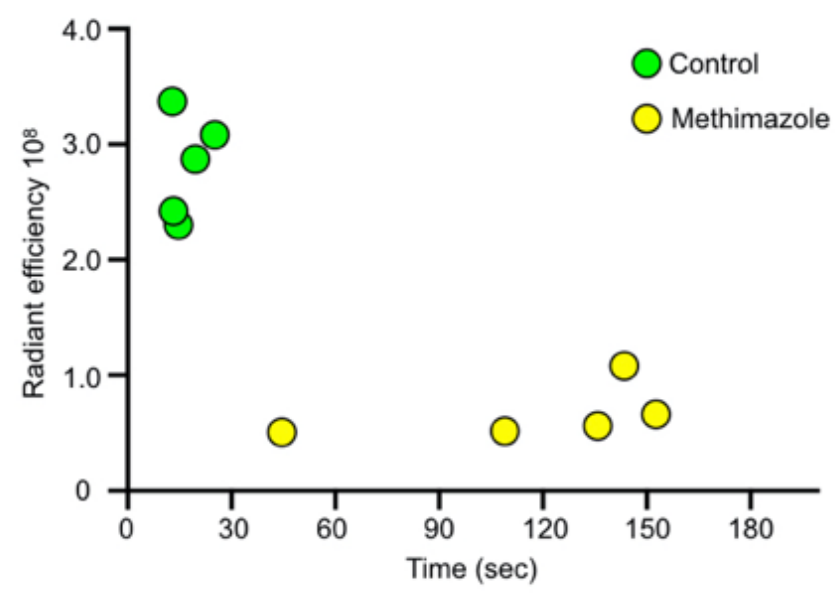

Figure 4. Buried food test. (a) Schematic illustrating mouse cage with a cookie buried in the upper right corner of the cage. (b) Bars show time (seconds) spent for mice treated with PBS $(n=5)$ or methimazole $(n=5)$ to find the buried food. (c) Correlation of radiant efficiency and time in food buried test.

\section{Discussion}

Here we demonstrate an objective diagnostic method for detection of smell disorders which is correlated to the degree of damage and can potentially guide the treatment. All current diagnostic methods are based on a smell perception of the afflicted patients and 
are subjective by design. Despite being standardized and validated, these methods are culturally specific and individually subjective [15]. Certain odors may not be familiar to those outside the geographic region where the test had been developed. Moreover, odor identification methods only can diagnose the presence of a problem, but not the site of the damage or etiology. The only existing method that can do both is a surgical biopsy. However, the invasive nature of the procedure limits its use in multiple ways.

We tested our imaging agent using a mouse model in which olfactory ablation was achieved via methimazole injections. In response to methimazole injection, within the same day several cell types in the olfactory epithelium, such as Bowman's gland cells, sustentacular cells, and OSN, display signs of apoptosis, followed by detachment of cells from the basal lamina [16]. This damage follows a similar sensorineural smell loss pattern seen in upper-respiratory viral infection or chronic inflammation due to seasonal allergies.

We observed loss of Nav1.7 channel expression following olfactory ablation using methimazole (Figure 1). The expression of Nav1.7 channels was substantially reduced in the OSN, and nerve bundles located in lamina propria., whereas it was less affected at the level of olfactory bulbs. The extent of Nav1.7 expresison (as measured by radiant efficiency of the Nav1.7 imaging agent) was inversely correlated with the time mouse spent finding food in a buried food test. One mouse that found the cookie in $44 \mathrm{~s}$, much faster compared to others from the olfactory-ablated group, likely found it randomly and mostly based on visual clues, rather than via smell. 
Further, we assessed the possibility of detecting COVID-19-related olfaction loss with our Nav1.7 imaging agent. COVID-19-related smell disorders are possibly caused by multiple mechanisms. There is a direct tropism of the virus to sustentacular and microvillar cells covering the OSN, inflammatory damage to OSN, as they are exposed to environmental factors, and inflammation involving focal mucosal swelling and obstruction to airways [17]. Together, these mechanisms cause diminished Nav channel expression in the OSN, and olfactory nerve bundles located in the nasal cavity as shown by IHC. This makes it possible to image and diagnose a smell disorder related to COVID-19 using our imaging agent.

As described above, it is crucial to develop a non-invasive, fast, and objective method to diagnose smell disorders. Intravenously injected Tsp1a-IR800 selectively accumulates in OSN and olfactory nerve bundles located in the nasal cavity. Existing flexible endoscopes from several medical technology companies (Stryker, Olymp etc.) can detect the IR wavelength emitted Tsp1a-IR800. Potentially, in any otolaryngologist office, a patient could be injected with Tsp1a-IR800 and be scoped at the level of the olfactory epithelium to confirm or exclude the smell disorder caused by either virus, COVID-19, or chronic inflammation. Due to the minimally invasive nature of the nasal cavity scope, the method can be used as a treatment monitoring tool, and in the development of therapeutic treatments. 


\section{Materials and methods}

\section{Synthesis of Tsp1a-IR800.}

Synthetic Tsp1a was prepared as in our previous report $[6,18]$. Tsp1a-IR800 was synthesized using Tsp1a peptide $\left(0.71 \mathrm{mM}, 250 \mu \mathrm{g}\right.$ in $100 \mu \mathrm{L}$ of $\left.\mathrm{H}_{2} \mathrm{O}\right)$, which was diluted in a 25-mM Tris-buffered aqueous solution (Supplementary Figure). To this reaction mixture, $20 \mu \mathrm{L}$ of a $50-\mathrm{mM}$ solution of L-ascorbic acid in $\mathrm{H}_{2} \mathrm{O}$ and $20 \mu \mathrm{L}$ of a 50 mM aqueous $\mathrm{CuSO}_{4}$ solution in $\mathrm{H}_{2} \mathrm{O}$, were added. This step was followed by the addition of $50 \mu \mathrm{g}(44 \mathrm{nmol})$ of IR800 azide in $50 \mu \mathrm{L}$ of acetonitrile, after which the reaction mixture was stirred at room temperature in the dark for $4 \mathrm{~h}$. The crude mixture was separated via reversed-phase (RP) HPLC, yielding $44 \mu \mathrm{g}(10 \mathrm{nmol} ; 26 \%)$ of Tsp1a800. The final analytical RP-HPLC purification showed $96 \%$ purity. LCMS-ES (ESI+), $\mathrm{m} / \mathrm{z}$ calculated for Tsp1a_Pra_IR800, $\left[\mathrm{C}_{207} \mathrm{H}_{293} \mathrm{~N}_{48} \mathrm{O}_{57} \mathrm{~S}_{10}\right] \quad 4682.87$, $\left[\mathrm{C}_{207} \mathrm{H}_{293} \mathrm{~N}_{48} \mathrm{O}_{57} \mathrm{~S}_{10}+3 \mathrm{H}\right]^{3+}$ 1563.09, found $\quad[\mathrm{M}+3 \mathrm{H}]^{3+}$ 1564.10, $\left[\mathrm{C}_{207} \mathrm{H}_{293} \mathrm{~N}_{48} \mathrm{O}_{57} \mathrm{~S}_{10}+4 \mathrm{H}\right]^{4+}$ 1172.00, found $\quad[\mathrm{M}+4 \mathrm{H}]^{4+}$ 1173.20, $\left[\mathrm{C}_{207} \mathrm{H}_{293} \mathrm{~N}_{48} \mathrm{O}_{57} \mathrm{~S}_{10}+5 \mathrm{H}\right]^{5+} 938.16$, found $[\mathrm{M}+5 \mathrm{H}]^{5+} 939.00$.

\section{Animal works}

All animal care and procedures were approved by the Animal Care and Use Committees (IACUC) of Memorial Sloan-Kettering Cancer Center. Hsd:athymic female mice Nude-Foxn1 ${ }^{\text {nu }}$ (6-8 weeks old) were used in the study. We first assessed the possibility of visualizing the olfactory nerve in normal mice using Tsp1a-IR800. Nine athymic nude mice were divided into three groups. The main group was intravenously injected with Tsp1a-IR800 (1 nmol, $100 \mu \mathrm{L}$ PBS), the control group was injected with 
phosphate-buffered saline (PBS), and a third group was injected with a combination of Tsp1a-IR800 and unmodified Tsp1a (blocking agent).

\section{Olfactory ablation}

Methimazole treatment was performed to ablate the olfactory nerve as described elsewhere [19]. Mice were injected with methimazole dissolved in isotonic saline (50 mg/kg body weight) via intraperitoneal administration, on days 0 and 3 . In vivo fluorescence imaging was performed on day 8 after the first injection. We have used 3 groups of mice, treated with methimazole, normal mice, and a blocking group. After imaging, all mice were sacrificed, and organs of interests were dissected.

\section{Behavioral experiment}

The buried food test was performed as described [20]. Briefly, mice were fasted overnight by removing all chow pellets from the cage 18-24 $\mathrm{h}$ before the test. On the test day, a mouse was transferred to the clean cage containing $3 \mathrm{~cm}$ bedding, with the hidden cookie in one of the random corners $2 \mathrm{~cm}$ deep. The observer retreats to the observation station and starts the timer and stops the stopwatch when the subject mouse finds the buried food (Figure 4a). A subject was considered to have uncovered the cookie when it starts to eat, usually holding the food with forepaws.

\section{Imaging}

All mice were anesthetized using an intraperitoneal injection of a ketamine/xylazine cocktail and in-vivo epifluorescence images obtained using an IVIS Spectrum imaging 
system (PerkinElmer) using filters set for $745 \mathrm{~nm}$ excitation and $810 \mathrm{~nm}$ emission. Animals were sacrificed and olfactory nerve/bulb, muscle, heart, spleen, kidney, and liver were removed and imaged ex vivo using the IVIS system. Autofluorescence was removed through spectral unmixing. Semiquantitative analysis of the Tsp1a-IR800 signal was conducted by measuring the average radiant efficiency (in units of $\left[\mathrm{p} / \mathrm{s} / \mathrm{cm}^{2} / \mathrm{sr}\right] /\left[\mu \mathrm{W} / \mathrm{cm}^{2}\right]$ ) in regions of interest (ROls) that were placed on the region of olfactory epithelium/bulb.

\section{Tabletop fluorescence microscopy}

Olfactory epithelium tissues of mice intravenously injected with PBS (control), Tsp1aIR800 or Tsp1a-IR800/Tsp1a formulation were stained with a solution $10 \mu \mathrm{g} / \mathrm{mL}$ Hoechst 33,342 in PBS and placed over a coverslip glass slide $(48 \times 60$ mm no. 1 thickness, Brain Research Laboratories, Newton, MA). Images were acquired using a laser scanning inverted-stand confocal microscope (Leica TCS SP8, Germany) using $725 \mathrm{~nm}$ laser excitation for IR800 (red) and $405 \mathrm{~nm}$ for Hoechst (blue).

\section{Dissection of olfactory bulb and epithelium}

Mice were anesthetized with ketamine/xylazine (100 and $20 \mu \mathrm{g} / \mathrm{g}$ body weight, respectively), perfused transcardially with $0.1 \mathrm{M}$ phosphate buffer (PB) followed by a PB buffered fixative containing 4\% paraformaldehyde (PFA) as described by Lin et al [21]. The dissection is divided into two components, the removal of the brain from the body and the removal of the skin and lower jaw. Under magnification, we removed the hard palate and the bones covering the brain, keeping nasal bones in place. The head was 
fixed in 4\% PFA overnight. Tissues were processed, decalcified and paraffin-embedded using a standard protocol.

\section{Immunohistochemistry and quantification of Nav1.7 expression}

Nav1.7 staining was performed at the Molecular Cytology Core Facility of MSK using a Discovery XT processor (Ventana Medical System, Tucson, AZ). We used an antiNav1.7 antibody [N68/6] (NeuroMab) that binds to both human and mouse Nav1.7 (0.5 $\mu \mathrm{g} / \mathrm{mL}$ ). Paraffin-embedded formalin-fixed $4-\mu \mathrm{m}$ sections were deparaffinized with EZPrep buffer. For IHC, a 3,3'-diaminobenzidine (DAB) detection kit (Ventana Medical Systems, Tucson, AZ) was used according to the manufacturer's instructions. Sections were counterstained with hematoxylin and eosin (H\&E) and coverslip using Permount (Fisher Scientific, Pittsburgh, PA).

Nav1.7 quantification was performed on digitalized slides. The threshold for signal intensity in the DAB (brown) and H\&E (blue, representing all tissue area) channels was determined via an automated script using ImageJ analysis software. Color deconvolution was used to separate blue and brown signals and the threshold values were kept constant: $0-114$ for $\mathrm{DAB}$ and $0-235$ for H\&E. The relative Nav1.7 positive area was calculated by dividing the brown (DAB) area by the blue (total tissue area).

\section{Statistical analysis}

Statistical analysis of Nav1.7 expression was performed using R v3.6.3 (R Core Team 2020, R Foundation for Statistical Computing, Vienna, Austria) and GraphPad Prism 9 
(GraphPad Software, San Diego, CA). A student $t$-test was used to compare Nav1.7 expression (ratio Nav1.7 expression/Total tissue area) between different groups of mice. Pearson correlation coefficient was used to examine the correlation between the time in food buried test and radiant efficiency. Results with a p-value of 0.05 were considered statistically significant. Data points represent mean values, and error bars represent standard deviations. 


\section{Declarations}

\section{Funding}

This work was supported by National Institutes of Health Grant Nos. R01 EB029769 (T.R. \& G.F.K.), R01 CA204441 (T.R.), K99 GM145587 (J.G.), and R01 CA204441$03 S 1$ (J.G.), the Australian National Health \& Medical Research Council (Principal Research Fellowship APP1136889 to G.F.K.) and the Australia Research Council (Centre of Excellence Grant CE200100012 to G.F.K.). The funding sources were not involved in study design, data collection and analysis, writing of the report, or the decision to submit this article for publication.

\section{Author Contributions.}

D.A., J.G., T.R., S.P. and N.P. conceived the study and designed the experiments. D.A. J.G, T.V., P.D.S.F, S.R., S.J, A.O., L.C. and T.R. carried out the experiments and collected the data. J.G., C.Y.C., G.K. and T.R. produced Tps1a-IR-800. D.A., N.P. and T.R. analyzed the data, D.A., J.G., N.P. and T.R. conducted statistical analysis of the data. D.A., J.G., N.P. and T.R. primarily wrote and edited the manuscript. All the authors reviewed and approved the manuscript.

\section{Disclosure of Potential Conflicts of Interest}

S.P. and T.R. are shareholders of Summit Biomedical Imaging, T.R. is now an executive of and shareholder in Novartis AG. J.G., P.D.S.F., G.K. and T.R. are co- 
inventors on a Tsp1a-related patent application. All other authors have no conflicts to declare.

\section{Acknowledgements}

The authors thank the support of Memorial Sloan Kettering Cancer Center's Animal Imaging Core Facility and Molecular Cytology Core Facility. We especially thank Eric Chan for the help with the Image J plugins for Nav1.7 quantifications. We also thank Terry Helms for creating illustrations. 


\section{References}

1. Bushdid, C., et al., Humans can discriminate more than 1 trillion olfactory stimuli. Science, 2014. 343(6177): p. 1370-2.

2. Leonardos, G., D. Kendall, and N. Barnard, Odor Threshold Determinations of 53 Odorant Chemicals. Journal of the Air Pollution Control Association, 1969. 19(2): p. 91-95.

3. Mombaerts, P., Seven-transmembrane proteins as odorant and chemosensory receptors. Science, 1999. 286(5440): p. 707-11.

4. Hoffman, H.J., et al., New chemosensory component in the US National Health and Nutrition Examination Survey (NHANES): first-year results for measured olfactory dysfunction. Reviews in Endocrine \& Metabolic Disorders, 2016. 17(2): p. 221-240.

5. Weiss, J., et al., Loss-of-function mutations in sodium channel $\mathrm{Na}(\mathrm{v}) 1.7$ cause anosmia. Nature, 2011. 472(7342): p. 186-190.

6. Gonzales, J., et al., Fluorescence Imaging of Peripheral Nerves by a Na(v)1.7-Targeted Inhibitor Cystine Knot Peptide. Bioconjugate Chemistry, 2019. 30(11): p. 2879-2888.

7. Gonzales, J., et al., Bimodal Imaging of Mouse Peripheral Nerves with Chlorin Tracers. Mol Pharm, 2021. 18(3): p. 940-951.

8. Mainland, J.D., et al., Identifying Treatments for Taste and Smell Disorders: Gaps and Opportunities. Chem Senses, 2020. 45(7): p. 493-502.

9. Temmel, A.F.P., et al., Characteristics of olfactory disorders in relation to major causes of olfactory loss. Archives of Otolaryngology-Head \& Neck Surgery, 2002. 128(6): p. 635641.

10. Kollndorfer, K., et al., Self-esteem as an important factor in quality of life and depressive symptoms in anosmia: A pilot study. Clin Otolaryngol, 2017. 42(6): p. 1229-1234.

11. Croy, I., S. Nordin, and T. Hummel, Olfactory disorders and quality of life--an updated review. Chem Senses, 2014. 39(3): p. 185-94.

12. Philpott, C.M. and D. Boak, The impact of olfactory disorders in the United kingdom. Chem Senses, 2014. 39(8): p. 711-8.

13. Sivam, A., et al., Olfactory Dysfunction in Older Adults is Associated with Feelings of Depression and Loneliness. Chem Senses, 2016. 41(4): p. 293-9.

14. Rupasinghe, D.B., et al., Localization of $\mathrm{Na}(\mathrm{v}) 1.7$ in the normal and injured rodent olfactory system indicates a critical role in olfaction, pheromone sensing and immune function. Channels, 2012. 6(2): p. 103-110.

15. Hummel, T., et al., Position paper on olfactory dysfunction. Rhinology, 2016. 56(1): p. 130.

16. Bergström, U., et al., Methimazole-induced damage in the olfactory mucosa: effects on ultrastructure and glutathione levels. Toxicol Pathol, 2003. 31(4): p. 379-87.

17. Mastrangelo, A., M. Bonato, and P. Cinque, Smell and taste disorders in COVID-19: From pathogenesis to clinical features and outcomes. Neurosci Lett, 2021. 748: p. 135694.

18. Gonzales, J., et al., Bimodal Imaging of Mouse Peripheral Nerves with Chlorin Tracers. Molecular Pharmaceutics, 2021. 18(3): p. 940-951.

19. Bergman, U., et al., Differential effects of olfactory toxicants on olfactory regeneration. Archives of Toxicology, 2002. 76(2): p. 104-112. 
20. Yang, M. and J.N. Crawley, Simple behavioral assessment of mouse olfaction. Curr Protoc Neurosci, 2009. Chapter 8: p. Unit 8.24.

21. Lin, W., et al., TRPM5-Expressing Solitary Chemosensory Cells Respond to Odorous Irritants. Journal of Neurophysiology, 2008. 99(3): p. 1451-1460.

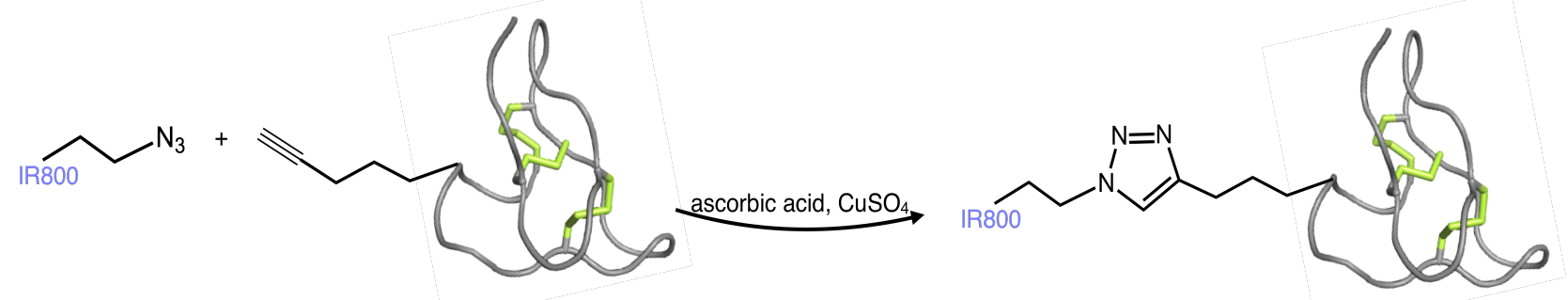

Supplementary Figure Synthetic pathway towards Tsp1a-IR800. The schematic chemical reaction for the formation of Tsp1a-IR800 where the IR800 fluorophore attached to an azido group reacts with a triple bond to afford the fluorescent imaging agent. 